\title{
Applications of Mathematical Modelling to Biological Pattern Formation
}

\author{
Philip K. Maini
}

Centre for Mathematical Biology, Mathematical Institute, Oxford, OX1 3LB, UK

\begin{abstract}
The formation of spatiotemporal patterning in biology has intrigued experimentalists and theoreticians for many generations. Here we present a brief review of some mathematical models for pattern formation and then focus on three models which use the phenomenon of chemotaxis to generate pattern.
\end{abstract}

\section{Introduction}

One of the characteristic features of nature is the enormous diversity of spatial structure and form present in plants and animals. Understanding how such patterns arise is the central goal of a large body of experimental and theoretical research. Although the spectacular advances over the past decade in molecular biology have resulted in detailed descriptions of the spatiotemporal dynamics of various genes and their products, such studies shed only very limited light on the underlying origin of these dynamics. The latter is the interest of the mathematical biologist, and many models have been proposed to describe the formation of biological patterns.

One of the most famous examples of spatiotemporal pattern formation is the Belousov-Zhabotinsky chemical reaction, in which bromate ions oxidise malonic acidic in a reaction catalysed by iron which, depending on its state, can assume two different colours - reddish-orange or blue. By observing the colour changes, one can see that this reaction exhibits a wide range of spatiotemporal patterning, such as propagating fronts, spiral waves, target patterns and toroidal scrolls. This reaction may be modelled mathematically by a coupled system of nonlinear partial differential equations which account for the reaction kinetics between species and also their diffusion. Solutions of the resultant system of equations exhibit the experimentally observed phenomena for chemically realistic parameter values (for review, see $[1,2]$ and references therein).

Such oscillatory and wave-like patterns also arise in physiology. For example, the heart beats in response to an electrical stimulus which moves in a wavelike fashion across the heart. This may be modelled phenomenologically by a system of equations which are not dissimiliar to those mentioned above, and the resultant models have been used to try to understand abnormal heart behaviour, such as fibrillation, which can lead to death (for review, see [3]). Spiral waves also play a key part in the life cycle of the slime mould Dictyostelium discoideum and we will focus on this in section 4 .

The above are examples of patterns that change in space and in time. Perhaps the most elegant model for temporally stationary spatial patterns is that 
proposed by Turing [4]. He showed that a pair of reacting and diffusing chemicals could evolve from initial nearly uniform steady state chemical concentration profiles to spatially varying profiles. He hypothesized that if one of the reacting pair of chemicals was a growth hormone, then this underlying spatially heterogeneous chemical profile would result in spatially non-uniform growth leading to the development of structure, or a spatial pattern. Hence he termed these chemicals morphogens.

Turing's analysis showed that it was possible to chose a reaction-diffusion system in which the kinetics yielded a steady state which was stable in the absence of diffusion. However, the introduction of diffusion, which we intuitively think of as a stablizing process, causes instability. Hence, this was termed diffusion-driven instability and is an example of self-organisation, or an emergent property.

Since Turing's seminal paper there has been an enormous amount of literature in which different types of nonlinear models are derived and analysed, all based on his original idea. Although the existence of morphogens still remains a controversial issue, there are a number of candidates (see, for example, [5]). Some 40 years after Turing predicted the phenomenon of diffusion-driven instability, it was observed in a chemical system, the Chloride-Iodide-Malonic Acid (or CIMA) reaction $[6,7]$.

In this paper we present a brief review of previous models that have been proposed to account for spatial patterning in biology. We then consider, in a little more detail, three different models which have the common underlying theme of involving chemotaxis, a widely-used mechanism for directed cell movement which has been extensively studied from both the experimental and theoretical viewpoints $[8,9]$.

\section{Models for Spatial Pattern Formation: A Brief Review}

A number of models have been proposed to account for spatial pattern formation and regeneration in several areas of developmental biology. This is now a huge area of research and to aim to review all such models in one short paper is too ambitious. Therefore, here we present only a few of the models in order to give the reader a flavour of the area.

Broadly speaking, most models may be classified as either chemical prepattern models or cell movement models. We consider each in turn:

(i) Chemical pre-pattern models: These models assume that a spatial pattern in some chemical (termed a morphogen) is set up and cells respond to this pattern by differentiating accordingly, that is, cells are distributed in a spatially uniform manner, and the spatial heterogeneity arises due to the information (termed positional information) cells receive from the chemical pre-pattern. There are two main ways in which such a spatially non-uniform pattern in chemical concentration may arise: It may be due to a simple source-sink mechanism, coupled with diffusion and degradation, which cells then interpret via a complex mechanism involving multiple thresholds [10], or it may arise due to a reaction-diffusion, or Turing system. Such a pattern, in its simplest one-dimensional form, consists of a series of peaks and troughs, which require only one threshold of cellular of a series of peaks and troughs, which require on cell differentiation. These models may be couched in terms of partial differential equations, or discretized and analysed as cellular automata [11].

(ii) Cell movement models: These models assume that a spatial pattern arises in cell density, and cells then differentiate in a density-dependent manner; for in thills in low example, those cells in high density aggregates differentiate while cells in low density areas remain undifferentiated. There are a number of ways in which cell agrregation can move in response to mechanical and chemical aggregation can occur. Cells can move in response to mechanical and che sites cues, such as gradients in certain chemical concentrations and/or adhesive sites in the extracellular material in which they move. A number of authors have studied these types of models and shown that they can lead to complex spatial patterns in cell density (see, for example, $[12-14]$ ).

Models formulated on the hypotheses that cells move to minimize energy have also been shown to give rise to pattern formation and cell sorting behaviour consistent with a number of experimental observations [15-18].

Chemical pre-pattern and cell movement models have been used to account for a wide range of pattern formation and morphogenetic phenomena, including for a wide rangetelal patterning in gastrulation, pattern formation and regulation in Hydra, skeletal patterning in the limb, animal coat markings, feather formation on dorsal chick skin, tooth primordia formation in alligators, to name but a few (see [19], and references therein, for further details).

The above models are based on very different biological hypotheses and it is still an area of controversy as to which is correct. Although it appears that in in alligators, salamanders and certain tions and of to cell movement, in many other cases a pattern of cell condense patterning events has not yet been resolved (due to technical difficulties) it is still not clear what is cause and what is effect [20]

Although the biology underlying these models are different, several of them are based on short-range activation, long-range inhibition, which is a genera are based on short-range activation, long-range inhibition, which is a general mechanism for generating spatial pattern. Mathematically, the reaction-diffusion models and several of the cell movement models are coupled systems of nonlinear partial differential equations. Due to their complexity, most mathematical analyses have thus far consisted of determining linear stability and primary bifurcation points. One finds that for both model types, the patterns produced in the vicinity of a primary bifurcation point are eigenfunctions of the Laplacian satisfying the appropriate boundary conditions. This can be confirmed by numerical simulation. Therefore, both model classes make similar patterning predictions. Althese types of models to distinguish between hypotheses, it does make it possible to make predictions that are independent of the underlying biological hypotheses. This has been exploited to derive certain developmental constraints [21]. Perhaps the best known of these is that spotted animals with striped tails are much more common than striped animals with spotted tails [2]. 
The above models consider pattern formation at a macroscopic level and therefore cannot account for patterning on a finer scale. In many developing tissues, adjacent cells diverge in character to create a fine-grained pattern of cells in contrasting states of differentiation. For example, in the developing nervous system, nascent neural cells inhibit their neighbours from becoming committed to a neural fate, resulting in a fine-grained differentiation pattern. This is an example of the general lateral inhibition principle that underlies many such patterns. The appropriate type of mathematical model in this case is a coupled system of ordinary differential equations characterising the temporal changes of key chemicals or proteins in each individual cell, with cells being coupled by nearest neighbour interactions [22].

\section{Primitive Streak Formation}

Many pattern formation and morphogenetic studies focus on aspects of avian development. Although there are detailed experimental descriptions of the events occurring in the early stages of chick development, less is known about the mechanisms that underly these events. One of the earliest pattern forming events, which also sets the stage for the proper coordination of subsequent development, is the formation of the primitive streak. During streak formation, a group of specialized cells at the posterior margin of the disc-like blastoderm moves anteriorly across the disc to about three-fifths the way across, and then it regresses. During regression, large scale cell movements occur turning the two-dimensional embryo into a three-dimensional structure. The movement of the streak is an example of a novel type of travelling wave. Travelling waves of constant speed and profile are well-known to occur in scalar reaction-diffusion equations and they have been applied to account for the propagation of a favoured gene through a population, while such waves in coupled systems have been used to account for the invasion of one species by another [2], or the closure of wounds [23].

To date, little in known about the mechanisms controlling progression and regression in the primitive streak. Recently, a simple model based on cell-chemotaxis was proposed and analysed, and shown to exhibit many of the features observed experimentally. Here, we briefly review the main points of the model and refer the reader to the original paper for full details [24].

The model postulates that the density $n(\mathrm{x}, \mathrm{t})$ at spatial position $\mathrm{x}$ and time $t$ of the subpopulation of cells that comprise the primitive streak secrete and respond to a chemoattractant which has concentration $u(\mathrm{x}, \mathrm{t})$ and takes the general form:

$$
\begin{aligned}
& \frac{\partial n}{\partial t}=D_{n} \nabla \cdot(\nabla n-n \chi(u) \nabla u)+f_{1}(n, u) \\
& \frac{\partial u}{\partial t}=D_{u} \nabla^{2} u+f_{2}(n, u)
\end{aligned}
$$

where $D_{n}$ and $D_{u}$ are constant diffusion coefficients and the second term on the right-hand side of the first equation models movement up the gradient of the chemical (chemoattraction) with chemotactic sensitivity. Modelling how cells respond to external signalling cues is a very active area of research at the moment. To date, most models are phenomenological, although recently it has been shown how rules of motion at the individual cell level may be translated, using the ideas of biased random walks, to the cell population level [25]. For simplicity, here we assume that the sensitivity is a constant $\chi_{0}$. Net cell and chemical production are modelled by the terms $f_{1}$ and $f_{2}$ respectively. It is known that cell motion during these early stages of development occurs on a faster timescale than cell division, so $f_{1}$ can be taken to be 0 . The chemical kinetics is chosen to be of the form:

$$
f_{2}(n, u)=g(n)-\beta n u-\delta u
$$

where $g(n)$ accounts for secretion of the chemical by cells, and is assumed to depend sigmoidally on $n$. (In a very simple case, it can be taken to be 0 for $n<n_{1}$, and a non-zero constant $\gamma$ for $n \geq n_{1}$ for some constant threshold cell density level $n_{1}$ ). The other two terms in the equation above model chemical degradation due to the cells and to natural decay.

The appropriate boundary conditions are zero flux. If the model is considered on a one-dimensional spatial domain, then appropriate initial conditions for $n$ are that $n(x, 0)=n_{i}$ for $x<x_{i}$ but zero otherwise. If the initial chemical concentration is taken to be spatially uniform and the model equations solved numerically, it is found that this simple model exhibits progression followed by regression. The mechanism underlying this behaviour is that the initial cell density at the origin degrades the chemical concentration creating a gradient, up which cells start to move. At some later stage, however, the chemical concentration ahead of the advancing cell wave drops to a value below that behind the wave, reversing the chemical gradient and hence cell motion. It is possible to determine the parameter space in which this behaviour will occur and it turns out to be a robust phenomenon. The time of reversal can be approximated in terms of the parameters of the model as can the speed.
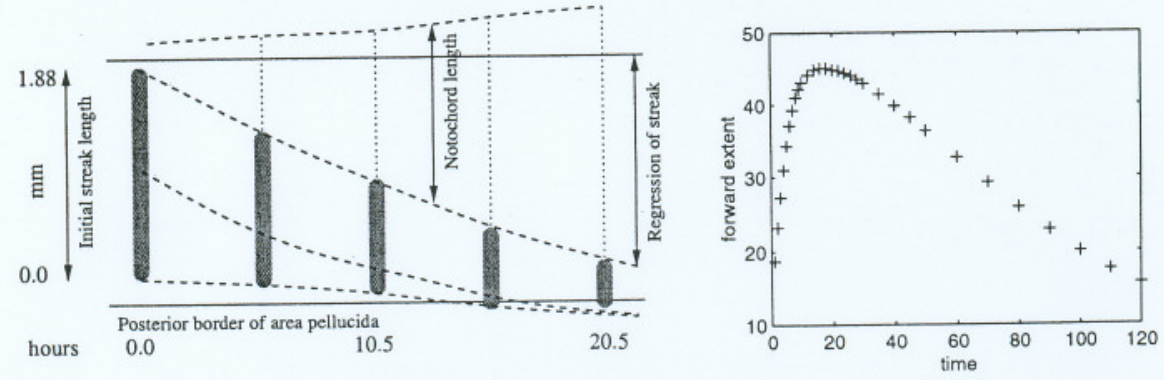

Fig. 1. Comparison of experimental data with numerical simulations of model equations (1)-(2) in one dimension showing the spatial extent of primitive streak regression Reproduced from [24] with permission. 
Simulation Initial Conditions
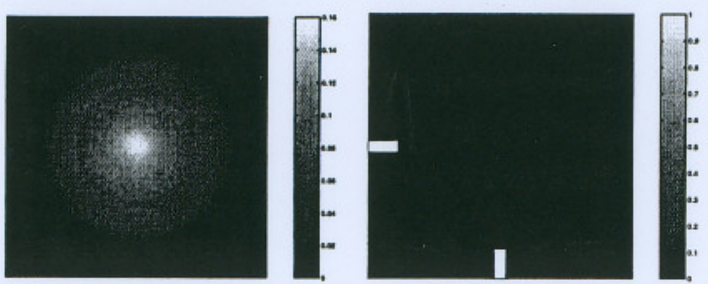

Simulation Initial Conditions
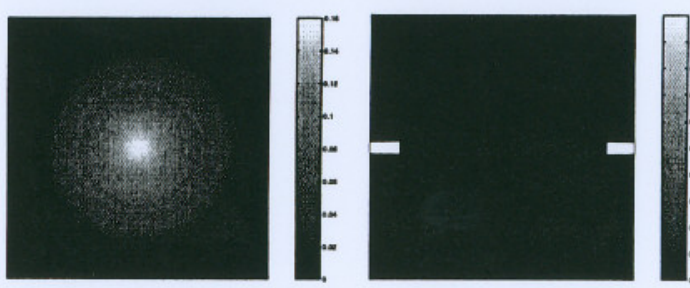

Prediction

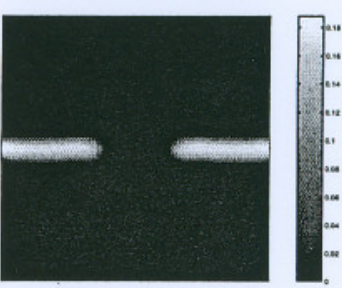

Simulation Initial Conditions
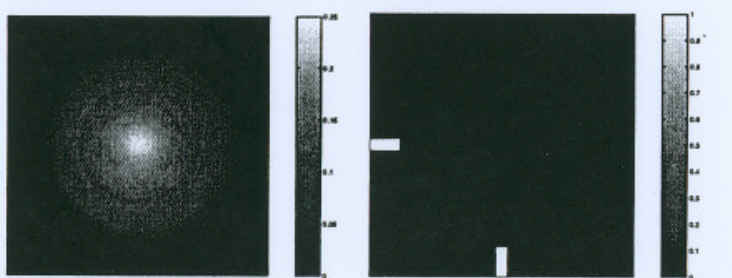

Prediction

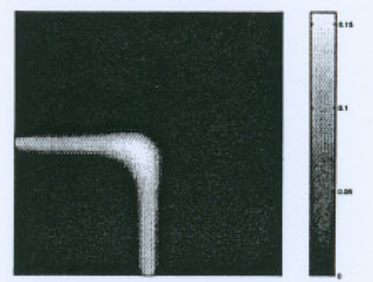

Fig. 2. Numerical simulations of model equations (1)-(2) for the case where a donor primitive streak is implanted at different places in a host. The host primitive streak is at the left-hand margin of the domain. The resulting "prediction" agrees with experimental observations. Note that two of the experiments give different predictions: the top panel predicts two ingrowing streaks, the bottom panel predicts fused streaks. The outcome of the numerical simulations is crucially dependent on the initial conditions and reflects the experimental observations that the outcome is either two separate streaks or a fused pair. The left-most panels show the concentration of chemoattractant. Reproduced from [24] with permission.

Of course, on a two-dimensional spatial domain, the above initial conditions result in a spreading disc of cells, whereas the primitive streak itself is a tightly compacted column of cells. By assuming that the initial chemical concentration on a two-dimensional domain has a maximum at the centre of the domain however, numerical simulations show that the model exhibits behaviour in close agreement with experimental observations (Figs 1 and 2). Fig. 3 shows how the model can be used to make experimentally testable predictions.

Hence, this simple model shows that the hypothesis that primitive streak formation is due to chemotaxis is a plausible one, and it makes experimentally testable predictions.

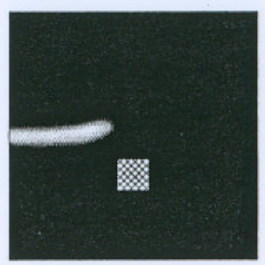

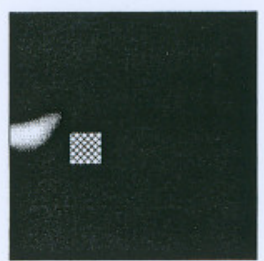

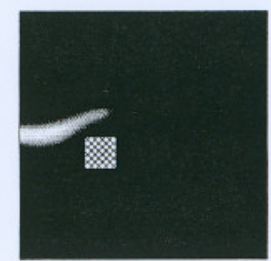

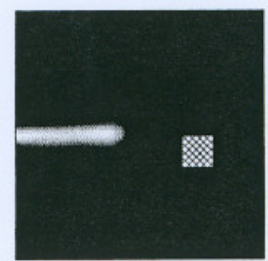

d
Fig. 3. Model predictions for streak development after experimental ablation (hatched area) of certain portions of the blastodisc. Reproduced from [24] with permission.

\section{Cell Aggregation in Slime Mould}

The amoebae of the cellular slime mould Dictyostelium discoideum (Dd) have a remarkable life cycle. In response to starvation conditions, the amoebae secrete a chemical, cyclic 3'5'-adenosine monophosphate (cAMP), which diffuses through the medium in the form of spiral waves. This is a chemottractant for the cells and causes the phenomenon of cell streaming, leading to cell aggregation and finally to the formation of a multicellular organism, known as the slug, composed typically of $10^{4}-10^{5}$ cells. In the body of the crawling slug, the cells differentiate into pre-spore and pre-stalk cells. Finally, the slug culminates into a fruiting body in which the stalk cells lift the spore cells, aiding their dispersal. The amoebae can remain in the spore stage for many years before emerging under favourable conditions.

This developmental program has been studied in great detail by experimentalists and theoreticians alike because it contains several elements, for example, signal propagation and tranduction, cell movement, sorting and differentiation, that are common to higher organisms. Hence it serves as an excellent model paradigm.

We briefly summarise the modelling here and refer the reader to [26-29] and references therein for full details. The molecular mechanisms of the cAMP signalling dynamics have been studied in detail and a number of models have been proposed. The simplest of these exploit the different time scales on which reactions occur to use a quasi-steady state assumption to reduce the order of 
the system, leading to a coupled pair of ordinary differential equations in which the variables are extracellular cAMP concentration and density of cAMP receptors. This is an excitable system and exhibits propagating spiral waves, in good agreement with those observed experimentally.

To model cell streaming, one must couple cell movement and chemotaxis to this excitable system. This may be done by considering the cells as discrete entities for which cAMP-dependent movement rules are specified, and the cAMP concentration profiles are determined by a numerically computed finite-difference approximation to the above excitable system. This method has the advantage of allowing one to follow individual cells and compare at a detailed level, the model with experimental observations. However, the form of the model is such that little mathematical insight can be gained. Alternatively, the cells can be modelled as a continuum. This has the disadvantage of allowing less detailed comparison with experiment, but the resultant system of coupled partial differential equations can be analysed mathematically to some extent [28]. We refer the reader to [29] for more details of these two alternative methods and a comparison between them.

Here, we consider the following continuum model $[26,27]$ :

$$
\begin{aligned}
& \frac{\partial n}{\partial t}=\nabla \cdot(\mu \nabla n-\chi(v) n \nabla u) \\
& \frac{\partial u}{\partial t}=\lambda\left[\phi(n) f_{1}(u, v)-(\phi(n)+\delta) f_{2}(u)\right]+\nabla^{2} u \\
& \frac{\partial v}{\partial t}=-g_{1}(u) v+g_{2}(u)(1-v),
\end{aligned}
$$

where $n, u$ and $v$ denote cell density, extracellular cAMP concentration and fraction of active cAMP receptors, respectively. The first equation is the cell conservation equation and incorporates diffusion, with cell diffusion coefficient $\mu$, and chemotaxis, with chemotactic sensitivity denoted by $\chi(v)$. The simplest form of $\chi(v)$, namely a constant, is not appropriate here, because it would mean that cells would move both in response to the front of the signalling cAMP wave and also in the waveback. This contradicts experimental observations, which show movement only in the wavefront. Moreover, it predicts that the net cell motion will be in the direction the signal is travelling, rather than in the direction from where the signal originated. This was known as the "chemotactic paradox" and can be resolved by accounting for adaptation. Hence, the chemotactic sensitivity is taken to be of the form $\chi(v)=\chi_{0} v^{m} /\left(A^{m}+v^{m}\right), m>1$, where $\chi_{0}$ and $A$ are positive constants. This functional form reflects the hypothesis that an appreciable chemotactic response requires a minimal fraction of active receptors, yet, for a large fraction of active receptors, the response saturates.

The second and third equations are a simplified version of the excitable model of the cAMP-cell receptor dynamics [30] modified by cell density effects. The first term on the right-hand side of the second equation is the cAMP production term and assumes that the rate of production per unit cell density is $f_{1}(u, v)$, where $f_{1}(u, v)=\left(b v+v^{2}\right)\left(a+u^{2}\right) /\left(1+u^{2}\right)$. This models autocatalytic cAMP production with saturation, mediated by cAMP binding to active receptors. The rate of cAMP degradation per cell is taken to be $f_{2}(u)=d u$. The cell density dependence is taken to be $\phi(n)=n /(1-\rho n /(K+n))$, while $\delta$ accounts for cAMP degradation in the absence of cells. The parameters $a, b, d, \rho, K, \delta$ and $\lambda$ are all positive constants.
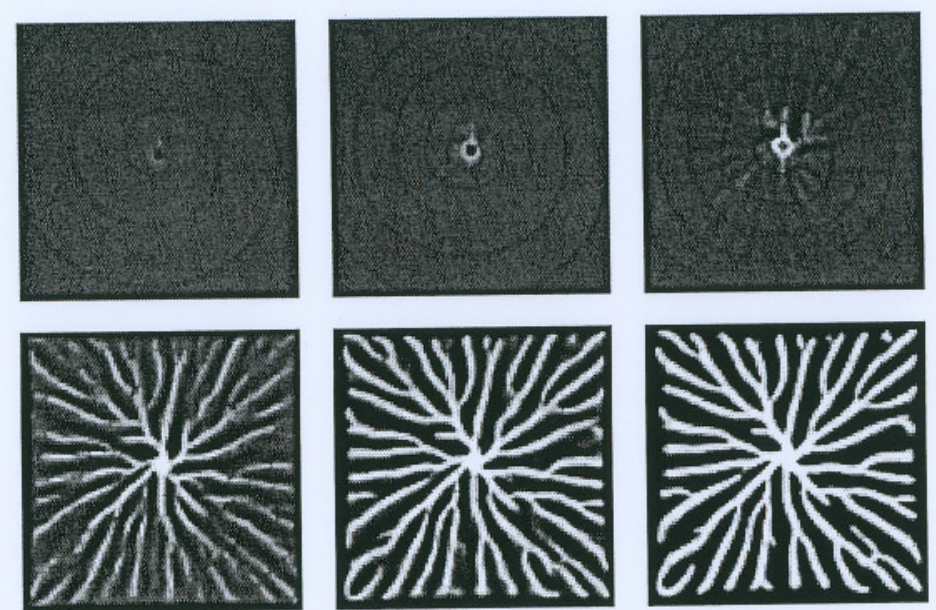

(a)
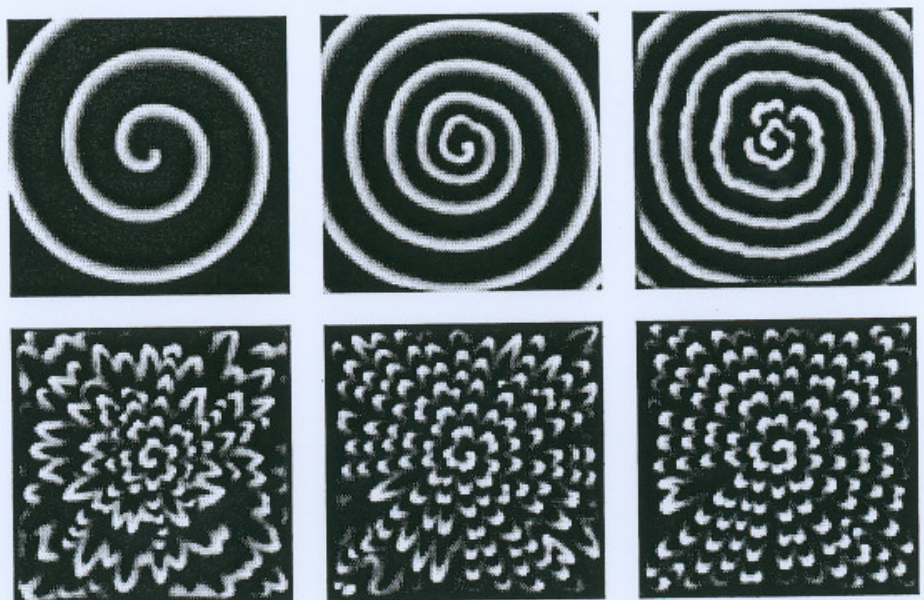

Fig. 4. Typical numerical simulation of model equations (4)-(6) showing the time evolution (left to right) of (a) cell density and (b) cAMP. Note the phenomenon of cell streaming (panels 5 and 6 in (a))

The first term on the right-hand side of the third equation accounts for receptor densitization and, assuming the law of mass action, $g_{1}(u)=k_{1} u$. The second 
term models resensitization of the desensitized $(1-v)$ fraction of receptors, at the constant rate $g_{2}(u)=k_{2}$. The parameters $k_{1}$ and $k_{2}$ are positive constants.

Good estimates of most of the parameters are available from the experimental literature and we refer the reader to the original paper [26] for these estimates and also for full details on the derivation of each term, which we have only sketched above. Substituting these parameter values into the model, we find that the model captures the key features of the cell streaming process. Fig. 4 shows a typical model simulation.

It is found that the initial uniform state is unstable from the outset, suggesting that the coupled dynamics of cAMP wave propagation and cell movement exhibit a patterning instability perpendicular to the direction of wave propagation. This has been investigated in an analytically-tractable caricature of the above model from which it is possible to derive explicit conditions on the parameters for the uniform steady state to go unstable, and to predict the wavelength of the fastest growing mode from the dispersion relation. The results are in good agreement with the numerical simulations of the full model above and experimental observations [27].

Numerical simulations show that low initial cell densities lead to the formation of a central hole agreeing with experimental observations in which the excitability of the medium is lower. It can be shown mathematically that, in the model, this is equivalent to lowering the cell density.

The model is not only consistent with a number of experimental observations, it can also provide alternative explanations for some observed phenomena. For example, it is known that the wavespeed and wavelength of the spiral patterns decrease as cell streaming procedes. This has been explained by assuming that complex biochemical changes must be occurring in the medium resulting in the parameters changing their values. However, the above model, in which the parameter values are kept constant, exhibits identical behaviour. It can be shown mathematically that this occurs due to the spiral dynamics.

\section{$5 \quad$ Pigmentation Patterning in Fishes}

A number of models have been proposed to account for the spectacular variety of pigmentation patterns seen on animals. These models show that the complexity of the pattern depends on the size of the domain at the instance when the pattern is laid down. None of these studies considered domain growth during the pattern formation process as it was assumed that, particularly in mammals, domain growth simply caused the pattern to change quantitatively, not qualitatively. However, it was shown recently that the pigmentation patterns on certain fishes change qualitatively with domain growth [31]. The authors studied the striped patterns on the marine angelfish, Pomacanthus, and observed that as the fish grows, its stripes grow wider apart until they are about twice the distance apart as in the juvenile case. At this stage, new stripes appear between the existing stripes in such a way that the original interstripe spacing is restored. The new stripes are thinner than the pre-existing stripes but they gradually broaden.
It was shown in [31] that this mode-doubling phenomenon was consistent with the Turing theory. The general Turing model takes the form:

$$
\begin{aligned}
& \frac{\partial u}{\partial t}=D_{u} \nabla^{2} u+f(u, v) \\
& \frac{\partial v}{\partial t}=D_{v} \nabla^{2} v+g(u, v)
\end{aligned}
$$

where $f$ and $g$ are the reaction kinetics for chemicals with concentrations $u$ and $v$. The authors considered very simple piecewise linear kinetics and included domain growth in an ad hoc way in their simulations. Their results showed that as the domain grew, new stripes were inserted in a fashion similar to that observed on the fish, except that the inserted stripes had exactly the same width as the pre-existing stripes. This is to be expected as the wavelength of the pattern in a Turing system depends only on the model parameters.

It is not yet clear if the pigmentation pattern in Pomacanthus arises due to a chemical pre-pattern (as hypothesized in this model) or due to cell movement. Observations in other fish, such as zebrafish, suggest that cell movement may play an important role in pigmentation patterning. Therefore, a model was developed in [32] which accounted for cell movement and modelled domain growth in a mathematically rigorous way. It was shown that, under domain growth, the concentration $c$ of a chemical in a reaction-diffusion system satisfies an equation of the form:

$$
\frac{\partial c}{\partial t}+\nabla \cdot(\underline{a} c)=D \nabla^{2} c+f(\underline{u})
$$

where $D$ is the diffusion coefficient, $\underline{a}$ is the velocity of growth of the domain and $\underline{u}$ is the vector of chemical concentrations. It can be shown that this system undergoes mode doubling for quite general forms of reaction kinetics and that this phenomenon can arise not only via insertion, but also by splitting [33].

Cell movement may be incorporated into this model by assuming that cell density, $n$, satisfies an equation of the form:

$$
\frac{\partial n}{\partial t}+\nabla \cdot(n \underline{a})=D_{n} \nabla^{2} n-\nabla \cdot\left[\chi_{u}(u) n \nabla u+\chi_{v}(v) n \nabla v\right]+n\left(r_{x}+r_{y}\right)
$$

where $a=\left(r_{x} x \mathbf{i}+r_{y} y \mathbf{j}\right)$ is the velocity field (assumed uniform for simplicity) generated by growth, $r_{x}$ and $r_{y}$ are growth rates in the $x$ and $y$ directions, respectively, $u$ and $v$ are the concentrations of the chemicals in the reactiondiffusion system, $\chi_{u}(u)$ and $\chi_{v}(v)$ are the chemotactic sensitivities. The last term on the right-hand side is a cell source term chosen to ensure conservation of cell density.

In [32] it is shown that not only can a model of this form produce mode doubling behaviour (obviously possible since the underlying Turing model is unchanged) but that, for biologically realistic parameters, the inserted stripes are narrower to begin with and then gradually broaden. The reason behind this behaviour is that although the newly inserted stripes of chemical concentration in the underlying reaction-diffusion system have width identical to the pre-existing 
stripes, the cell rearrangement induced by this new pattern occurs slowly, for realistic diffusion coefficients. As a result, the new inserted stripes in cell density are thinner than the existing stripes, broadening only gradually (Fig. 5). In fact, it can be shown that for suitable choice of chemotactic sensitivites $\chi_{u}(u)$ and $\chi_{v}(v)$, this model can give rise to cell density steady state patterns of thick and thin stripes [34].

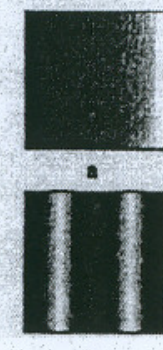

c

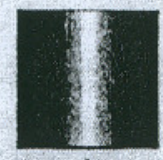

b

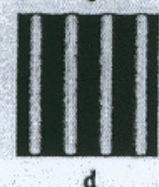

d
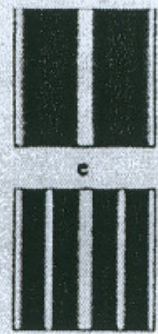
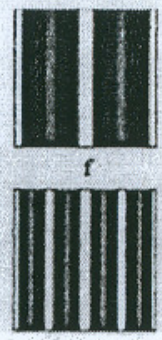

h
Fig. 5. Cell density plots in a numerical simulation of the fish pigmentation mode (equation (10) plus corresponding Turing system) showing stripe insertion as the domain grows (a) - (h). For convenience the domain has been rescaled to a fixed size. Reproduced from [34] with permission.

\section{Discussion}

The models presented in sections 3-5 share the common underlying process of chemotaxis but give rise to very different patterns due to the different dynamics that generate the pattern in chemoattractant. In the model for primitive streak a simple gradient in chemoattractant concentration is set up via degradation and diffusion. In the case of the slime mould, spiral waves of cAMP concentration are the result of the cAMP-active cAMP receptor system being an excitable medium, while in the fish pigmentation model, the chemical patterns are set up via diffusion-driven instability. The latter model also incorporated domain growth. One of the main future challenges in this general area is the detailed study of growth. In the model presented, growth was prescribed, for simplicity. In actual fact, of course, growth will result from the internal dynamics and this presents a new modelling challenge [35].

It is clear that biology offers mathematics a wide variety of novel and challenging problems. It is natural to ask what mathematics offers biology. Most biological processes interact in a nonlinear way. Therefore, to forecast the consequences of these interactions one must use a language in which nonlinearities can be manipulated. At the moment, mathematics is the only language that can do so. Therefore mathematics can be used to test hypothesis and also to make experimentally testable predictions. The three models presented in sections $3-5$ illustrate how mathematics may be used in this way.

\section{References}

1. B.R. Johnson, S.K. Scott: Chemical Soc. Reviews, 25, 265 (1996)

2. J.D. Murray: Mathematical Biology, 2nd edn. (Springer-Verlag 1993)

3. A.V. Panfilov, A.V. Holden (eds): Computational Biology of the Heart, (John Wiley \& Sons, Chichester 1997)

4. A.M. Turing: Phil. Trans. Roy. Soc. Lond. B 327, 37-72 (1952)

5. L.G. Harrison: Kinetic Theory of Pattern Formation, (Cambridge University Press, 1993)

6. V. Castets, E. Dulos, J. Boissonade, P. De Kepper: Phys. Rev. Lett., 64, 2953 (1990)

7. Q. Ouyang, H. L. Swinney: Nature (London) 352, 610 (1991)

8. J. P. Armitage, J.M. Lackie: Biology of the Chemotactic Response, (Cambridge University Press 1990)

9. W. Alt, A. Deutsch, G. Dunn (eds): Dynamics of Cell and Tissue Motion, (Birkhäuser 1997)

10. L. Wolpert: J. theor. Biol., 25, 1 (1969)

11. J.B.L. Bard: J. theor. Biol., 93, 363 (1981)

12. E.F. Keller, L.A. Segel: J. theor. Biol., 30, 235 (1971)

13. P.K. Maini, M.R. Myerscough, K.H. Winters, J.D.Murray: Bull. Math. Biol., 53, 701 (1991)

14. G.F. Oster, J.D. Murray, A.K. Harris: J. Embryol.exp. Morph., 78, 83 (1983)

15. M.S. Steinberg: J. exp. Zool., 173, 395 (1970)

16. D. Sulsky, S. Childress, J.K. Percus: J. theor. Biol., 106, 275 (1984)

17. G. Cocho, R. Pérez-Pascual, J.L. Rius: J. theor. Biol., 125, 419 (1987)

18. G. Cocho, R. Pérez-Pascual, J.L. Rius, F. Soto: J. theor. Biol., 125, 437 (1987)

19. P.K. Maini: 'Mathematical models for biological pattern formation'. In: On Growth and Form: Spatio-temporal Patterning in Biology. ed. by M.A.J. Chaplain, G.D Singh, J.C. McLachlan (John Wiley \& Sons, Chichester 1999) pp. 111-128

20. P.K. Maini, M. Solursh: Int. Rev. Cytology, 129, 91 (1991)

21. G.F. Oster, J.D. Murray: Zool., 251, 186 (1989)

22. J.R. Collier, N.A.M. Monk, P.K. Maini, J.H. Lewis: J. theor. Biol., 183, 429 (1996)

23. P.D. Dale, P.K. Maini, J.A. Sherratt: Math. Biosciences 124, 127 (1994)

24. K.J. Painter, P.K. Maini, H.G. Othmer: Bull. Math. Biol. 62, 501 (2000)

25. H.G. Othmer, A. Stevens: SIAM J. Appl. Math. 57, 1044 (1997)

26. T. Höfer, J.A. Sherratt, P.K. Maini: Proc. Roy. Soc. Lond. B 259, 249 (1995)

27. T. Höfer, J.A. Sherratt, P.K. Maini: Physica D 85, 425 (1995)

28. T. Höfer, P.K. Maini: Phys. Rev. E 56, 2074 (1997)

29. J.A. Sherratt, J.C. Dallon, T. Höfer, P.K. Maini: 'Mathematical modelling of signalling in Dictyostelium discoideum'. In Microbial Signalling and Communication. ed. by R. England, G. Hobbs, N. Bainton, D. McL. Roberts (Cambridge University Press 1999) pp. 241-254

30. J.-L. Martiel, A. Goldbeter: Biohys. J. 52, 807 (1987)

31. S. Kondo, R. Asai: Nature 376, 765 (1995)

32. K.J. Painter, P.K. Maini, H.G. Othmer: Proc. Natl. Acad. Sci. 96, 5549 (1999)

33. E.J. Crampin, E.A. Gaffney, P.K. Maini: Bull. Math. Biol. 61, 1093 (1999)

34. K.J. Painter, P.K. Maini, H.G. Othmer: J. Math. Biol. (accepted)

35. R. Dillon, H.G. Othmer: J. theor. Biol. 197, 295 (1999) 
D. Reguera L.L. Bonilla J.M. Rubí (Eds.)

\section{Coherent Structures in Complex Systems}

Selected Papers of the XVII Sitges Conference on Statistical Mechanics

Held at Sitges, Barcelona, Spain, 5-9 June 2000 


\section{Lecture Notes in Physics}

\section{Editorial Board}

R. Beig, Wien, Austria

J. Ehlers, Potsdam, Germany

U. Frisch, Nice, France

K. Hepp, Zürich, Switzerland

W. Hillebrandt, Garching, Germany

D. Imboden, Zürich, Switzerland

R. L. Jaffe, Cambridge, MA, USA

R. Kippenhahn, Göttingen, Germany

R. Lipowsky, Golm, Germany

H. v. Löhneysen, Karlsruhe, Germany

I. Ojima, Kyoto, Japan

H. A. Weidenmüller, Heidelberg, Germany

J. Wess, München, Germany

J. Zittartz, Köln, Germany

\section{Springer}

Berlin

Heidelberg

New York

Barcelona

Hong Kong

London

Milan

Paris

Singapore

Tokyo

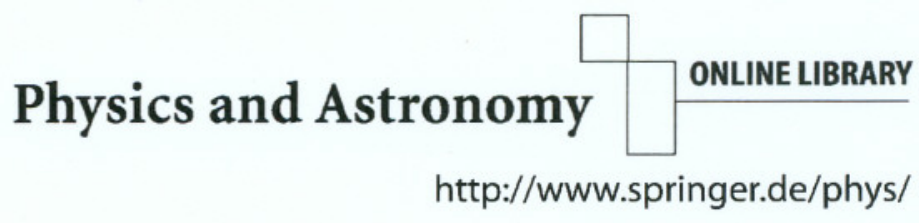




\section{Editors}

David Reguera

José Miguel Rubí

Department de Física Fonamental, Universitat de Barcelona

Diagonal 647, Barcelona 08028, Spain

Luis López Bonilla

Departamento de Matemáticas

Escuela Politécnica Superior

Universidad Carlos III de Madrid

Avenida de la Universidad 30, Leganés 28911, Spain

Library of Congress Cataloging-in-Publication Data applied for.

Die Deutsche Bibliothek - CIP-Einheitsaufnahme

Coherent structures in complex systems : selected papers of the XVII

Sitges Conference on Statistical Mechanics, held at Sitges, Barcelona,

Spain, 5 - 9 June 2000 / D. Reguera ... (ed.). - Berlin ; Heidelberg ;

New York; Barcelona ; Hong Kong ; London ; Milan ; Paris ; Singapore

; Tokyo : Springer, 2001

(Lecture notes in physics; Vol. 567)

(Physics and astronomy online library)

ISBN 3-540-41705-2

ISSN $0075-8450$

ISBN 3-540-41705-2 Springer-Verlag Berlin Heidelberg New York

This work is subject to copyright. All rights are reserved, whether the whole or part of the material is concerned, specifically the rights of translation, reprinting, reuse of illustrations, recitation, broadcasting, reproduction on microfilm or in any other way, and storage in data banks. Duplication of this publication or parts thereof is permitted only under the provisions of the German Copyright Law of September 9, 1965, in its current version, and permission for use must always be obtained from Springer-Verlag. Violations are liable for prosecution under the German Copyright Law.

Springer-Verlag Berlin Heidelberg New York

a member of BertelsmannSpringer Science+Business Media GmbH

http://www.springer.de

(C) Springer-Verlag Berlin Heidelberg 2001

Printed in Germany

The use of general descriptive names, registered names, trademarks, etc. in this publication does not imply, even in the absence of a specific statement, that such names are exempt from the relevant protective laws and regulations and therefore free for general use.

Typesetting: Camera-ready by the authors/editors

Camera-data conversion by Steingraeber Satztechnik GmbH Heidelberg

Cover design: design \& production, Heidelberg

Printed on acid-free paper

SPIN: 10797201

$57 / 3141 / \mathrm{du}-543210$ 